\title{
Determination of the carrier concentration in CdSe crystals from the effective infrared absorption coefficient measured by means of the photothermal infrared radiometry
}

\author{
M. Pawlak
}

Received: 21 July 2014/ Accepted: 27 October 2014/Published online: 11 November 2014

(C) The Author(s) 2014. This article is published with open access at Springerlink.com

\begin{abstract}
In this paper, a non-contact method that allows to determine the carrier concentration in CdSe crystals is presented. The method relies on the measurement of the effective infrared absorption coefficient by means of the photothermal infrared radiometry (PTR). In order to obtain the effective infrared absorption coefficient and thermal diffusivity, the frequency characteristics of the PTR signal were analyzed in the frame of a one-dimensional heat transport model for infrared semitransparent crystals. The carrier concentrations were estimated using a theory introduced by Ruda and a recently proposed normalization procedure for the PTR signal. The deduced carrier concentrations of the investigated CdSe crystals are in reasonable agreement with those obtained using Hall measurements and infrared spectroscopy. The method presented in this paper can also be applied to other semiconductors with the carrier concentration in the range of $10^{14}-10^{17} \mathrm{~cm}^{-3}$.
\end{abstract}

\section{Introduction}

Electrical characterization techniques of bulk semiconductors using conventional methods are very often inconvenient as electrical contacts are needed. For industrial applications, on-line inspections are required. In this context, non-contact, non-destructive and fast methods for the measurement of thermal and electrical properties of semiconductors seem to be more appropriate. Photothermal

\section{Pawlak $(\square)$}

Faculty of Physics, Astronomy and Informatics, Institute of Physics, Nicolaus Copernicus University, ul. Grudziądzka 5/7, 87-100 Toruń, Poland

e-mail: mpawlak@fizyka.umk.pl radiometry (PTR) is a non-contact method which enables to measure optical and thermal parameters of materials [1, 2]. In the case of semiconductors, PTR additionally yields information about the recombination parameters [3, 4]. Recently, it was found that for infrared (IR) semitransparent semiconductors, it is possible to obtain information on the carrier concentration with quantitative values after calibration to Hall carrier concentration [5]. In this paper, a simple normalization procedure of the PTR spectra, which allows to determine the carrier concentration in CdSe single crystals using the normalized effective IR absorption coefficient measured by means of the PTR method and the theory proposed by Ruda [6], is presented.

\section{Materials preparations and experimental methods}

\subsection{Materials}

n-type CdSe single crystals were grown by the high-pressure Bridgman method without a seed under an argon overpressure. The crystals were cut perpendicular to the growth direction into 0.9 - to 1.3 -mm-thick plates. Next, the plates were mechanically polished and chemically etched in a mixture of $\mathrm{K}_{2} \mathrm{Cr}_{2} \mathrm{O}_{7}, \mathrm{H}_{2} \mathrm{SO}_{3}$ and $\mathrm{H}_{2} \mathrm{O}$ in the proportion $3: 2: 1$. Then, they were treated in $\mathrm{CS}_{2}$ and hot $50 \% \mathrm{NaOH}$ solution and finally rinsed in water and ethyl alcohol.

\subsection{Experimental setups}

The PTR experimental setup is presented in Fig. 1. The thermal waves were excited using an argon ion laser (LASER) with an output power of $200 \mathrm{~mW}$ and a photon energy of $2.41 \mathrm{eV}$ (the operating wavelength $\lambda=514 \mathrm{~nm}$ ). The laser beam of about $2 \mathrm{~mm}$ diameter was intensity 
Fig. 1 PTR experimental setup

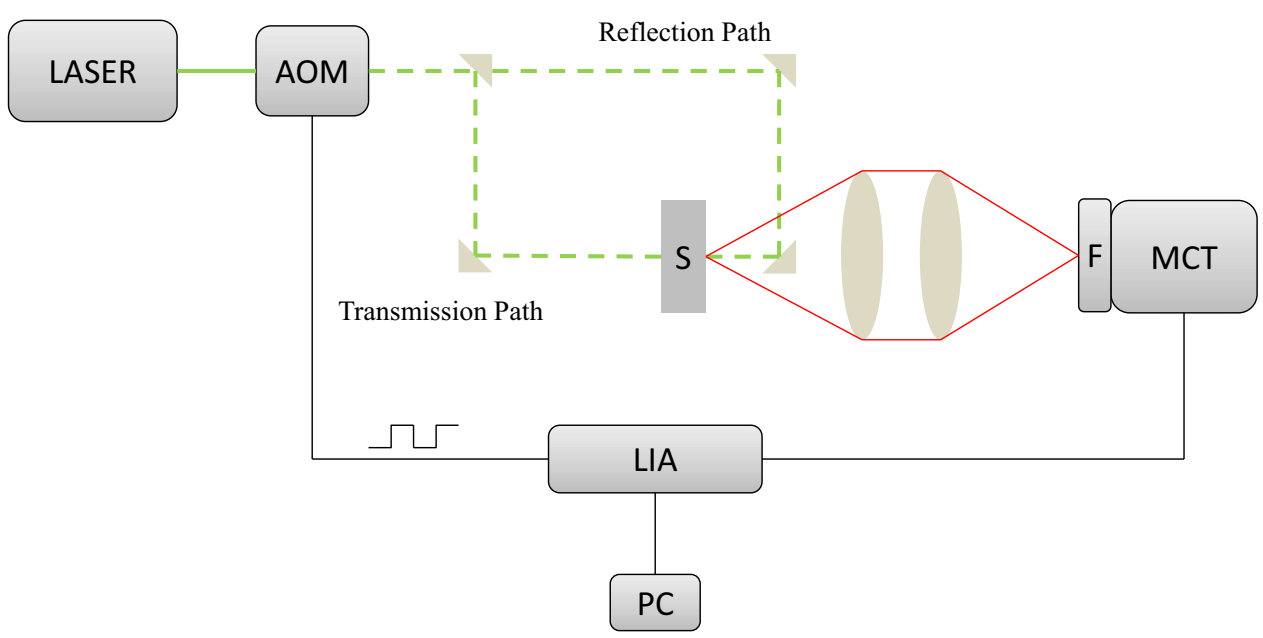

modulated by means of an acousto-optical modulator (A-O $\mathrm{M})$ in the frequency range $1 \mathrm{~Hz}-100 \mathrm{kHz}$ and focused onto the sample. Two $\mathrm{BaF}_{2}$ lenses $\left(\mathrm{L}_{1}, \mathrm{~L}_{2}\right)$ were used to collect IR radiation from the sample (S) on the photoconductive mercury cadmium telluride (MCT, $\mathrm{HgCdTe}$ ) detector with a detectivity peak at $10.6 \mu \mathrm{m}$. The IR transmittance of the $\mathrm{BaF}_{2}$ lens was about $90 \%$. The MCT detector covering the IR range from 2 to $12 \mu \mathrm{m}$ was supplied with a ZnSe cutoff filter $(\mathrm{F})$ in the front having a transmittance of about $90 \%$ in the wavelength range of the detector. The signal (amplitude $A$ and phase $\varphi$ ) of the MCT detector was amplified and filtered by a lock-in amplifier (LIA, Stanford 830 DSP) and analyzed by a personal computer (PC). The IR spectra were measured using the IRScope II, attached to a IFS 66 IR spectrometer (Bruker GmbH, Ettlingen, Germany) equipped with the liquid $\mathrm{N}_{2}$ cooled mercury cadmium telluride (MCT) detector. The detailed description of the IR spectrometer and an experimental procedure were presented elsewhere [7]. The IR absorption coefficients were calculated from the transmission spectra under the assumption that the optical reflection coefficient is constant in the investigated spectral range.

\section{Theoretical model}

In the Drude theory, the free carrier absorption is proportional to the carrier concentration $N$ and can be written as follows [8]

$\beta(\lambda)=\frac{e^{2} \cdot N \cdot \lambda^{2}}{4 \cdot \pi^{2} \cdot m_{\mathrm{e}} \cdot \varepsilon_{0} \cdot c^{3} \cdot n_{r} \cdot \tau}$,

where $e$ is the elementary charge, $m_{e}$ is the effective mass of an electron, $\varepsilon_{0}$ is the electric permittivity, $c$ is the speed of light and $\tau$ is the relaxation time of the scattering processes. For semiconductors, however, the dependence of

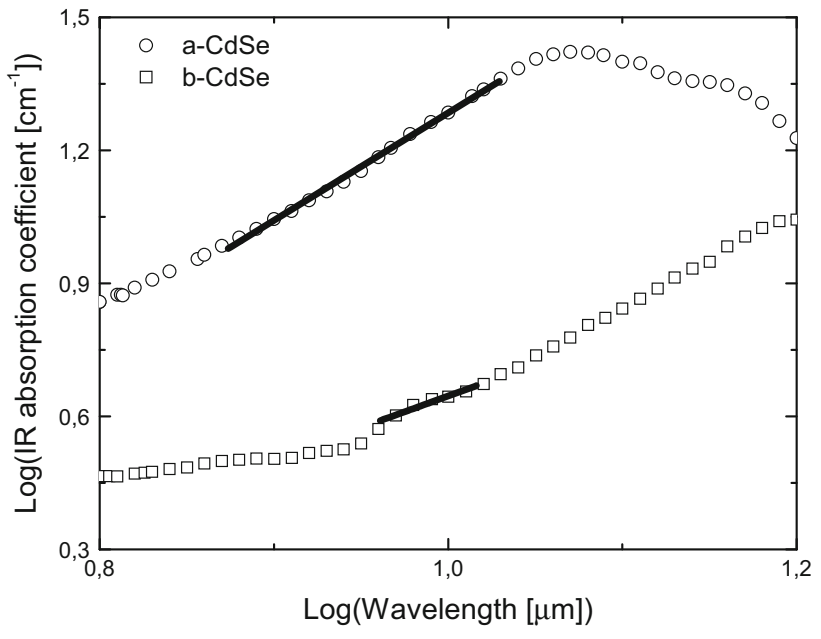

Fig. 2 IR absorption spectra as a function of wavelength $\lambda$ for $\mathrm{CdSe}$ crystals with the best fits using formula (4) (lines) to the experimental data. For clarity, the experimental points were reduced

the optical absorption coefficient on the wavelength of the absorbed radiation is rather of the type of $\beta(\lambda) \sim \lambda^{p}$, where $2<p \leq 3.5$. The parameter $p$ depends on the scattering mechanisms. There are three main types of scattering mechanisms: acoustic phonons, optical phonons and impurity scattering mechanisms. Using the quantum mechanical approach, it is possible to calculate the free carrier absorption coefficient. The IR absorption coefficient can be written as a sum of all scattering mechanisms as follows [9]

$\beta(\lambda)=A_{\text {acoustic }} \times \lambda^{1.5}+A_{\text {optical }} \cdot \lambda^{2.5}+A_{\text {impurity }} \cdot \lambda^{3.5}$,

where $A_{\text {acoustic }}, A_{\text {optical }}, A_{\text {impurity }}$ are proportionality coefficients for particular scattering mechanisms.

For semiconductors with the carrier concentration $N$, in the range of $10^{14}-10^{17} \mathrm{~cm}^{-3}$, Ruda [6] found the following 
relationship between the IR absorption coefficient measured at $\lambda=10 \mu \mathrm{m}$, the $p$ coefficient and the carrier concentration for $\mathrm{n}-\mathrm{ZnSe}$ :

$N=6.7 \cdot 10^{15} \cdot(3.5-p) \cdot \beta(\lambda), \quad \lambda=10 \mu \mathrm{m}$.

\section{Experimental results and discussion}

According to the validity range of the Ruda formula, the method presented should be applicable for the carrier concentration in the range $10^{14}-10^{17} \mathrm{~cm}^{-3}$. Two samples that possess a concentration within almost this range were available for this study. Figure 2 presents the IR absorption coefficients for CdSe crystals. As one can observe with an increasing excitation wavelength, the IR absorption coefficient increases monotonically. This behavior is typical for an absorption caused by free carriers. In Fig. 1, the experimental data are displayed together with a fit using the relation:

$\log (\beta(\lambda))=a+p \cdot \log (\lambda)$

The obtained fitting parameters $p$ for a-CdSe and b-CdSe are 2.43 and 2.30, respectively. The absolute error in determination of the $p$ parameter is $\Delta p=0.02$ and 0.03 , respectively. This means that the dominating scattering mechanism in these crystals is the phonons scattering mechanism. Assuming that the formula (3) can be applied to the n-CdSe crystals, the carrier concentration can be estimated. The carrier concentrations for a-CdSe and b-CdSe crystals calculated with the formula (3) are $1.38 \times 10^{17}$ and $3.54 \times 10^{16} \mathrm{~cm}^{-3}$, respectively.

The effective IR absorption coefficient and the thermal diffusivity of CdSe crystals were estimated using the onedimensional heat transport model for IR semitransparent crystals [5]. The experimental procedure consists in measuring the PTR signal in the transmission and reflection configurations with the PTR signal obtained in reflection configuration used as the reference signal. The applied theoretical approach is valid only for semiconductors with very short recombination lifetime (with a direct energy bandgap). For semiconductors with an indirect energy bandgap, such as silicon, the influence of plasma waves has to be taken into account. In paper [5], authors found the very good agreement between the experimental values of the thermal diffusivity obtained using the PTR method and the photopyroelectric technique [10]. The good agreement of the data obtained by the two different techniques proved the reliability of the PTR data and of the theoretical approach based on the adjustment of the two parameters, i.e., the thermal diffusivity $D_{\mathrm{t}}$ and the effective IR optical absorption coefficient $\beta_{\text {IR,eff. The obtained results of the effective IR absorption }}$ coefficient for a-CdSe and b-CdSe crystals were $7.5 \pm 0.2$ and $0.9 \pm 0.1 \mathrm{~cm}^{-1}$, respectively.

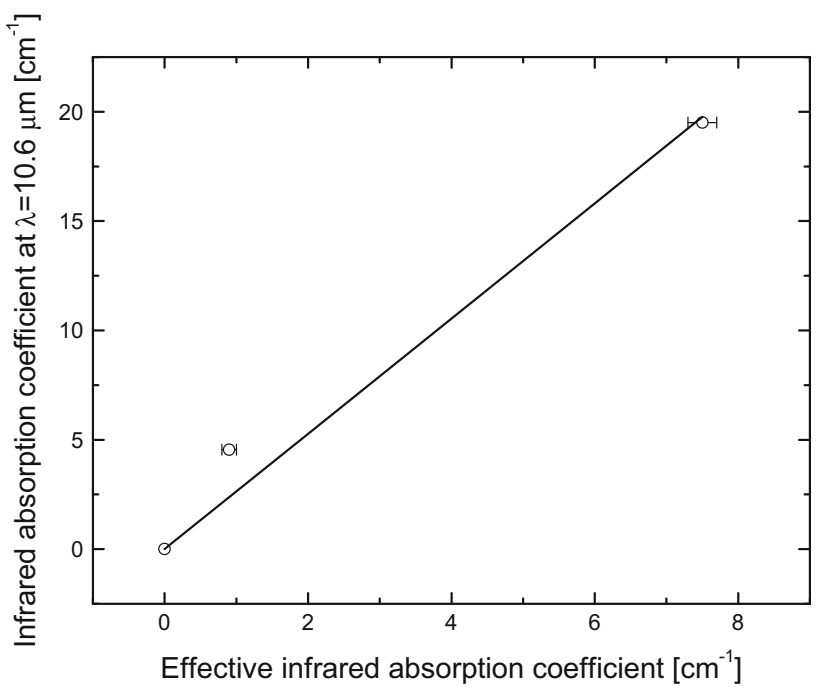

Fig. 3 Relation between the effective IR absorption coefficient and the IR absorption coefficient measured at $\lambda=10.6 \mu \mathrm{m}$ using the IR spectrometer

The effective IR absorption coefficient $\beta_{\mathrm{IR}, \text { eff }}$ represents the IR absorption coefficient averaged over the wavelength range of the MCT detector, in this case, in the wavelength between 2 and $12 \mu \mathrm{m}$. When the IR absorption coefficient increases monotonically along with the increasing excitation wavelength in the detection wavelength range, one can normalize the effective absorption coefficient to the IR absorption coefficient measured at $\lambda=10.6 \mu \mathrm{m}$. Figure 3 presents a proposed normalization procedure.

Figure 3 shows that the relation between the effective IR absorption coefficient and the IR absorption coefficient measured at $\lambda=10.6 \mu \mathrm{m}$ using the IR spectrometer for the samples investigated can be linear in the first approximation. The slope coefficient is $2.6 \pm 0.2$. A limitation of this procedure is worth to be discussed here. In the case of semiconductors with the carrier concentration higher than $2 \times 10^{17} \mathrm{~cm}^{-3}$, this procedure failed. For example, for $\mathrm{Cd}_{1-\mathrm{x}} \mathrm{Mg}_{\mathrm{x}} \mathrm{Se}$ crystals, the free carrier absorption region moves toward smaller wavelengths of about $6 \mu \mathrm{m}$ [7]. Multiplying the values of the effective IR absorption coefficient of CdSe crystals and the slope coefficient, one can calculate the normalized effective absorption coefficient $\beta_{\mathrm{n}, \mathrm{IR}, \mathrm{eff}}$. Assuming that the Ruda formula can be rewritten (3) as follows

$N=6.7 \cdot 10^{15} \cdot(3.5-p) \cdot \beta_{\mathrm{n}, \mathrm{IR}, \mathrm{fff}}$,

the carrier concentration in CdSe crystals can be calculated. Table 1 presents the values of the carrier concentration obtained using Hall measurements, the IR spectroscopy and the PTR method.

As one can see in Table 1, the carrier concentrations obtained using the three techniques for a-CdSe are in very 
Table 1 Carrier concentration in CdSe crystals measured using the Hall measurements, IR spectroscopy (IR) and PTR method

\begin{tabular}{lcccl}
\hline \multicolumn{4}{l}{ Carrier concentration } & $\left(10^{16} \mathrm{~cm}^{-3}\right)$ \\
\hline Sample & Hall & IR & PTR & Error (PTR) \\
\hline a-CdSe & 14.0 & 13.8 & 14.00 & \pm 0.80 \\
b-CdSe & 3.02 & 3.54 & 1.98 & \pm 0.61 \\
\hline
\end{tabular}

Also, the estimated errors of the data deduced from the PTR measurements are given

good agreement. However, for b-CdSe, the value obtained from the PTR experiment does not match with the other values within the experimental uncertainty. The estimated errors shown in Table 1 were obtained using the exact differential method taking into account errors in determination of the normalization slope, $p$ coefficients and effective IR absorption coefficients. Compared to the Hall method, the results obtained are in reasonable agreement. Although the Hall method usually has smaller errors (1-2\%), the error strongly depends on the sample preparation procedure (contacts) [11]. The PTR method presented in this paper does not suffer from the sample preparation procedure.

\section{Conclusions}

A simple normalization procedure of the IR spectra is proposed which allows to determine the carrier concentration in CdSe crystals using the effective IR absorption coefficient deduced from PTR experiments has been presented in this paper. The results obtained are in reasonable agreement with the results obtained from the Hall measurement and the IR spectroscopy. The proposed method can be used for IR semitransparent semiconductors and with the carrier concentration in the range between $10^{14}$ and $10^{17} \mathrm{~cm}^{-3}$.

Acknowledgments Author thanks Prof. J. Pelzl for the experimental support and many helpful discussions.

Open Access This article is distributed under the terms of the Creative Commons Attribution License which permits any use, distribution, and reproduction in any medium, provided the original author(s) and the source are credited.

\section{References}

1. R. Fuente, E. Apinaniz, A. Mendioroz, A. Salazar, J. Appl. Phys. 110, 033515 (2011)

2. M. Pawlak, M. Maliński, Infrared Phys. Technol. 64, 87-90 (2014)

3. A. Salnick, A. Mandelis, H. Ruda, C. Jean, J. Appl. Phys. 82, 1853 (1997)

4. M. Pawlak, M. Maliński, Opto-Electron. Rev. 22(1), 31-35 (2014)

5. M. Pawlak, M. Maliński, F. Firszt, J. Pelzl, A. Ludwig, A. Marasek, Meas. Sci. Technol. 25, 035204 (2014)

6. H.E. Ruda, J. Appl. Phys. 61, 3035-3043 (1987)

7. M. Pawlak, M. Maliński, F. Firszt, S. Łęgowski, H. Męczyński, J. Ollesch, A. Ludwig, A. Marasek, C. Schulte-Braucks, Infrared Phys. Technol. 64, 115-118 (2014)

8. P.K. Basu, Theory of Optical Processes in Semiconductors (Oxford Univeristy Press, New York, 1997)

9. J. Singh, I.-K. Oh, S. O. Kasap, Optical absorption, photoexcitation and excitons in solids: fundamental concepts, in PhotoExcited Processes, Diagnostics and Applications, Fundamentals and Advanced Topics, ed. by A. Peled, Kluwer (Academic Publishers, Boston, 2004), pp. 25-55

10. M. Pawlak, F. Firszt, S. Łęgowski, H. Męczyńska, J. Gibkes, J. Pelzl, Int. J. Thermophys. 31, 187 (2010)

11. L.J. van der Pauw, Philips Tech. Rev. 20, 220 (1958) 